\title{
Characterization of a human tumorsphere glioma orthotopic model using magnetic resonance imaging
}

\author{
Kelvin Wong • Geoffrey S. Young • Milan Makale • \\ Xintao Hu • Nalan Yildirim $\cdot$ Kemi Cui • \\ Stephen T. C. Wong $\cdot$ Santosh Kesari
}

Received: 16 September 2010/Accepted: 20 December 2010/Published online: 15 January 2011

(C) The Author(s) 2011. This article is published with open access at Springerlink.com

\begin{abstract}
Magnetic resonance imaging (MRI) is the imaging modality of choice by which to monitor patient gliomas and treatment effects, and has been applied to murine models of glioma. However, a major obstacle to the development of effective glioma therapeutics has been that widely used animal models of glioma have not accurately recapitulated the morphological heterogeneity and invasive nature of this very lethal human cancer. This deficiency is being alleviated somewhat as more representative models are being developed, but there is still a clear need for relevant yet practical models that are well-characterized in terms of their MRI features. Hence we sought to chronicle the MRI profile of a recently developed, comparatively straightforward human tumor stem cell (hTSC) derived
\end{abstract}

Kelvin Wong, Geoffrey S. Young, and Milan Makale-co-first authors.

K. Wong $\cdot$ X. Hu · N. Yildirim · K. Cui · S. T. C. Wong $(\bowtie)$ Division of Medical Physics, Department of Radiology, The Methodist Hospital-Weill Cornell Medical College and Center for Biotechnology and Informatics, The Methodist Hospital Research Institute, 6565 Fannin Street,

Brown 05-22, Houston, TX 77030, USA

e-mail: stwong@tmhs.org

M. Makale · S. Kesari ( $\square)$

Division of Neuro-Oncology, Department of Neurosciences, UC San Diego, Moores Cancer Center,

3855 Health Sciences Drive, La Jolla, CA 92093, USA

e-mail: skesari@ucsd.edu

\section{G. S. Young}

Harvard Medical School, Boston, MA, USA

\section{G. S. Young}

Division of Neuroradiology, Department of Radiology, Brigham and Women's Hospital, 75 Francis Street, Boston, MA 02115, USA glioma model in mice using conventional MRI methods. This model reproduces the salient features of gliomas in humans, including florid neoangiogenesis and aggressive invasion of normal brain. Accordingly, the variable, invasive morphology of hTSC gliomas visualized on MRI duplicated that seen in patients, and it differed considerably from the widely used U87 glioma model that does not invade normal brain. After several weeks of tumor growth the hTSC model exhibited an MRI contrast enhancing phenotype having variable intensity and an irregular shape, which mimicked the heterogeneous appearance observed with human glioma patients. The MRI findings reported here support the use of the hTSC glioma xenograft model combined with MRI, as a test platform for assessing candidate therapeutics for glioma, and for developing novel MR methods.

Keywords Tumor stem cell - Glioma .

Experimental tumor model · Permeability · Infiltration .

Angiogenesis · MRI

\section{Introduction}

A major obstacle to the development of effective glioma therapeutics and imaging agents is that until very recently, murine models of glioma have not accurately recapitulated the heterogeneous and highly invasive nature of this lethal form of brain cancer [1-3]. Even though these models have limitations, they are widely used, and in longitudinal studies the tumors are usually tracked with quantitative fluorescence/bioluminescence imaging [4, 5]. Optical methods do not capture tumor morphology, but magnetic resonance imaging (MRI), which has been applied to xenograft models, is able to acquire detailed images of 
implanted tumors [6, 7]. There is a clear need for the combination of a truly representative glioma model, and the imaging detail provided by MRI, to generate more relevant assessments of candidate glioma therapeutics [1]. Accordingly we sought to characterize the MRI features of a comparatively new murine model of glioma [8]. This model is an orthotopic xenograft using human glioma stem cells (hTSCs) that has been recently described-it closely reproduces the biology of glioma in humans, and it is straightforward to implement [8]. Our goal was to analyze conventional MRIs acquired longitudinally over several weeks to fully chronicle the behavior of the hTSC model, and thus create a glioma model/MRI—based test platform.

A rapidly evolving focus in glioma research is to identify agents that can suppress the invasive character of the disease [9]. Widely used glioma models such as the U87, U251 and U373 human glioma cell lines implanted in the frontal cortex of mice, while very useful for some types of studies, grow mainly by enlargement of the tumor mass, and do not exhibit infiltrative and invasive growth $[1,10$ 13]. While the recognized need for new glioma models has elicited the creation of genetic models which effectively recapitulate the features of patient tumors, these models create tumors in a variable time scale and only in a small proportion of the animals and are thus not entirely practical $[1,6,7,14]$. Another strategy for establishing in vivo test platforms is to transplant murine glioma neurospheres into the brains of immunocompromised (SCID) mice [15]. A recent variant of this approach utilizes human tumor stem cells (hTSC) implanted into the mouse brain, and has been shown to quite closely recapitulate the genotypic and phenotypic features of the original human tumor [13]. There are several reasons why such models should be characterized in terms of their MRI features. First, MRI is the modality of choice to routinely monitor tumor progress in glioma patients, so in mice the tumor response to a specific therapy can most directly be related to what might be observed in patients by using the same imaging modality [6]. In addition, MRI allows imaging of unlabeled cells, while optical methods require the tumor cells to stably express a fluorophore protein which might bias tumor cell behavior [16]. Finally, MRI is a non-invasive and accurate method by which tumor growth and volume can be determined in live mice.

Our proposed test platform is intended to facilitate MR brain imaging and contrast agent development. Although robust contrast enhancement is the clinical MRI marker of high tumor grade, up to $45 \%$ of non-enhancing lesions prove to have high grade histology at biopsy, and a small but significant number (4\%) of gliomas lack contrast enhancement [17-23]. Equally significantly, the correlation between histopathologic tumor grade and time to progression and survival is imperfect [21-23]. A large number of advanced techniques are under study in this context. Improvement in the reproducibility and sensitivity of routine contrast enhanced imaging might be facilitated using a cost-effective, practical, and relevant xenograft model.

In view of the foregoing, we sought to determine if the mouse hTSC model displayed the salient MRI findings of human glioma, which include obvious contrast enhancement and a surrounding irregular infiltrative morphology along the interface with the adjacent white matter [17-19]. Accordingly we characterized the MR imaging properties of the hTSC and U87 models in vivo using conventional MRI techniques. We applied these methods serially over several weeks to chronicle the progression of glioma as would be done during therapeutic agent development. The results illustrate that the hTSC-MRI mouse model recapitulates the MR findings diagnostic of human glioma and is a suitable platform for preclinical testing of new therapeutic and imaging approaches.

\section{Materials and methods}

Mouse xenograft models

\section{Animal subjects}

SCID mice of either sex and weighing between 20 and $25 \mathrm{~g}$ were housed in micro-isolator cages with autoclaved bedding, and supplied with autoclaved food and water. Proper procedures for SCID mouse handling, husbandry and attention to comfort and clinical condition after tumor implantation were strictly observed. The entire, detailed study protocol was reviewed and approved by the institutional review committee for animal research [Brigham and Women's Hospital (BWH) IACUC].

\section{Cell lines}

The U87 cell line was grown as an adherent culture in media containing serum. Primary high grade human glioma (glioblastoma) tissue was acquired surgically, dissociated, and cultured in stem cell selection media to form tumorspheres.

\section{Patient derived glioma cells}

The tumor tissue was freshly acquired during surgery performed before the patient underwent any chemo- or radiotherapy. The patient had a typically contrast enhancing mass upon MRI examination prior to surgery. Pathology examination of the tissue confirmed that the tumor was a primary glioblastoma. Following acquisition the tumor tissue was immediately dissociated in artificial CSF. The 
glioblastoma cells were plated into dishes containing stem cell isolation media and after 5 days the fully formed tumorspheres were dissociated and the cells implanted into mice [8].

\section{Intracranial implantation}

Aseptic surgical methods were followed in accordance with BWH guidelines. The U87 cultures and the tumorspheres (hTSC) were dissociated, counted, and concentrated into a compact suspension (1-2 $\mu \mathrm{l})$. The mice were anesthetized with ketamine/xylazine, the head was swabbed with Betadine ${ }^{\circledR}$, and body temperature was maintained with a circulating water pad. Tumor cell suspensions were injected stereotaxically into the right frontal cortex at $1 \mu \mathrm{l} /$ min using a $5 \mu$ l Hamilton syringe [21]. The syringe was left in place for $5 \mathrm{~min}$ before retraction to prevent reflux of the injected material. One group of mice $(n=7)$ was injected with approximately 1,500 hTSCs. A second group $(n=6)$ was implanted with 10,000 U87 cells; a relatively larger number of these cells was needed in order to establish viable tumors which approximated the size of the hTSC gliomas in a similar time frame.

\section{Longitudinal imaging studies}

\section{Imaging schedule}

MRI imaging of U87 and hTSC tumors was performed every week in U87 mice starting at week 3 and every 2 weeks in hTSC mice starting at week 4 . This was the imaging schedule that we found best balanced tumor viability with growth rates, which varied with tumor size at various timepoints. The hTSC tumor was not detectable by imaging until week 4 and it grew rapidly thereafter. The growth rate between U87 tumors was relatively uniform between subjects, and the mice were sacrificed after the second scan (week 2), while the hTSC mice were imaged between 2 and 5 times (weeks 2-5) before sacrifice, reflecting the greater heterogeneity of hTSC tumors in terms of growth rate.

\section{MR imaging protocol}

The mice were anesthetized with a mixture of $1.5 \%$ isofluorane and $95 \%$ oxygen throughout imaging. MRI was performed on a $4.7 \mathrm{~T}$ horizontal bore system interfaced with a commercial scanner console (Bruker BioSpin Corporation, MA) using a homogenous quadrature birdcage head coil. Coronal T2-weighted images (T2WI) (TR/ $\mathrm{TE}_{\text {eff }}=2,000 / 72 \mathrm{~ms}$ ) were acquired with a $2 \mathrm{D}$ fast spin echo sequence at $1 \mathrm{~mm}$ slice thickness with $80 \mu \mathrm{m} \times 130 \mu \mathrm{m}$ in-plane resolution. Magnevist ${ }^{\mathrm{Tm}} \mathrm{Gd}-$

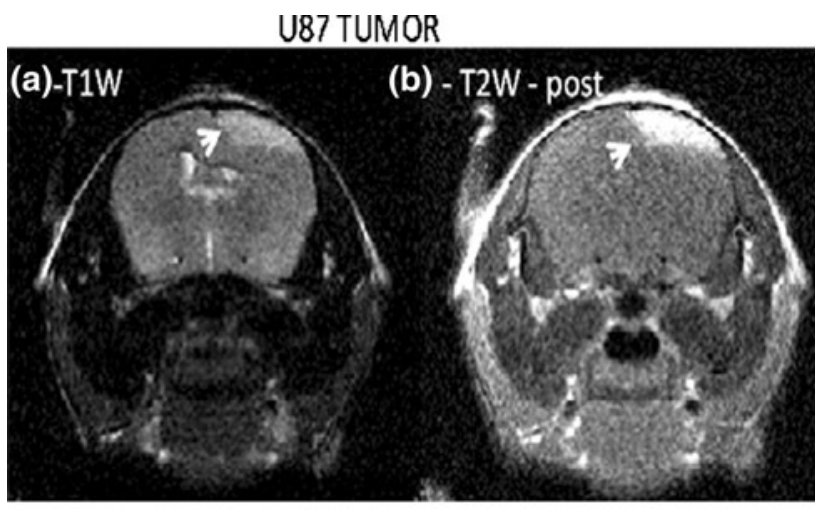

hTSCTUMOR

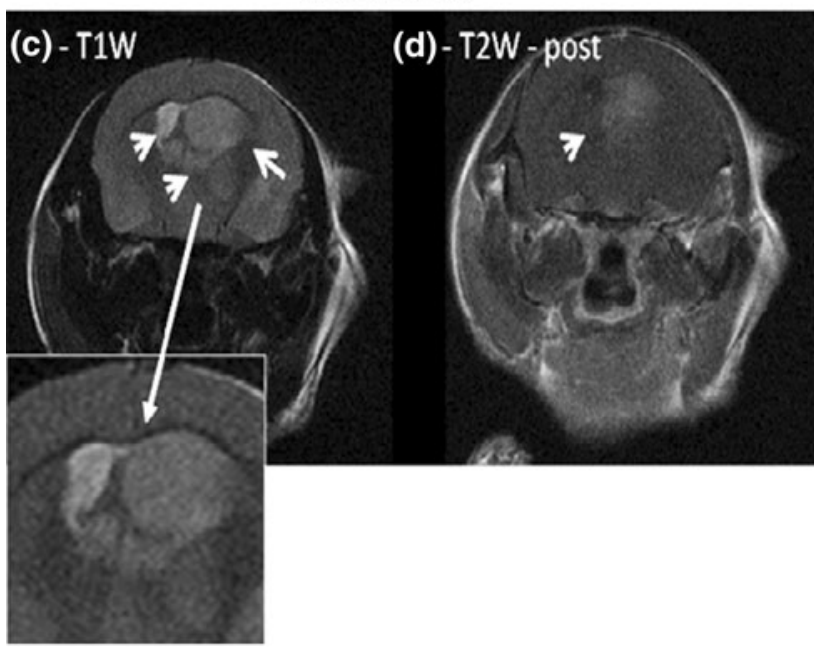

Fig. 1 MRI features of 2 week U87 and hTSC tumors. a U87 tumor $\mathrm{T} 2 \mathrm{~W}$ acquisition. Note the smooth outline of the U87 tumor (arrow). b Shows the post-contrast T1W image for the same tumor/slice as in panel (a). The tumor can be seen to be clearly enhancing. c T2W image slice of hTSC tumor. Note the irregular tumor outline (arrows), invasion of ventricular areas, non uniform shading, and an invasive profile on the left of the main mass. The expanded view of the tumor indicates the irregular and invasive nature of the hTSC tumor. d Postcontrast T1W image slice for same tumor as in panel (c). The hTSC tumor displays minimal contrast enhancement

DTPA was injected intraperitoneally at $0.7 \mathrm{mmol} / \mathrm{kg}$ and coronal $\mathrm{T} 1$-weighted images $(\mathrm{T} 1 \mathrm{WI})\left(\mathrm{TR} / \mathrm{TE}_{\text {eff }}=417 /\right.$ $24.5 \mathrm{~ms}$ ) were acquired once before and twice after contrast injection. Post-contrast imaging was performed at 12 min after injection, which based on our prior experience ensured optimal contrast enhancement (data not shown).

Data analysis

\section{MR tumor morphology}

A trained, blinded observer who was unaware of the origins of each MR acquisition, evaluated the MR volumes in terms of how well circumscribed the tumor boundary appeared and in terms of the presence or absence of an irregular infiltrative zone. 
Fig. 2 Histology of U87 and hTSC tumors. All images H\&E, $t$ tumor, $b$ normal brain. a U87 tumor appears rounded (darker staining regions) with distinct boundaries. b U87 tumor image clearly shows a typical rounded and cleanly demarcated tumor profile. c Closer view of U87 tumor shows very little infiltration of adjacent normal brain. d Overview of hTSC tumor showing highly scattered and invasive tumor with irregular outline (tumor includes darker staining areas) e hTSC tumor invasion into subventricular zone along ventricles. f hTSC tumor cell infiltrate into brain with no clear brain-tumor boundary
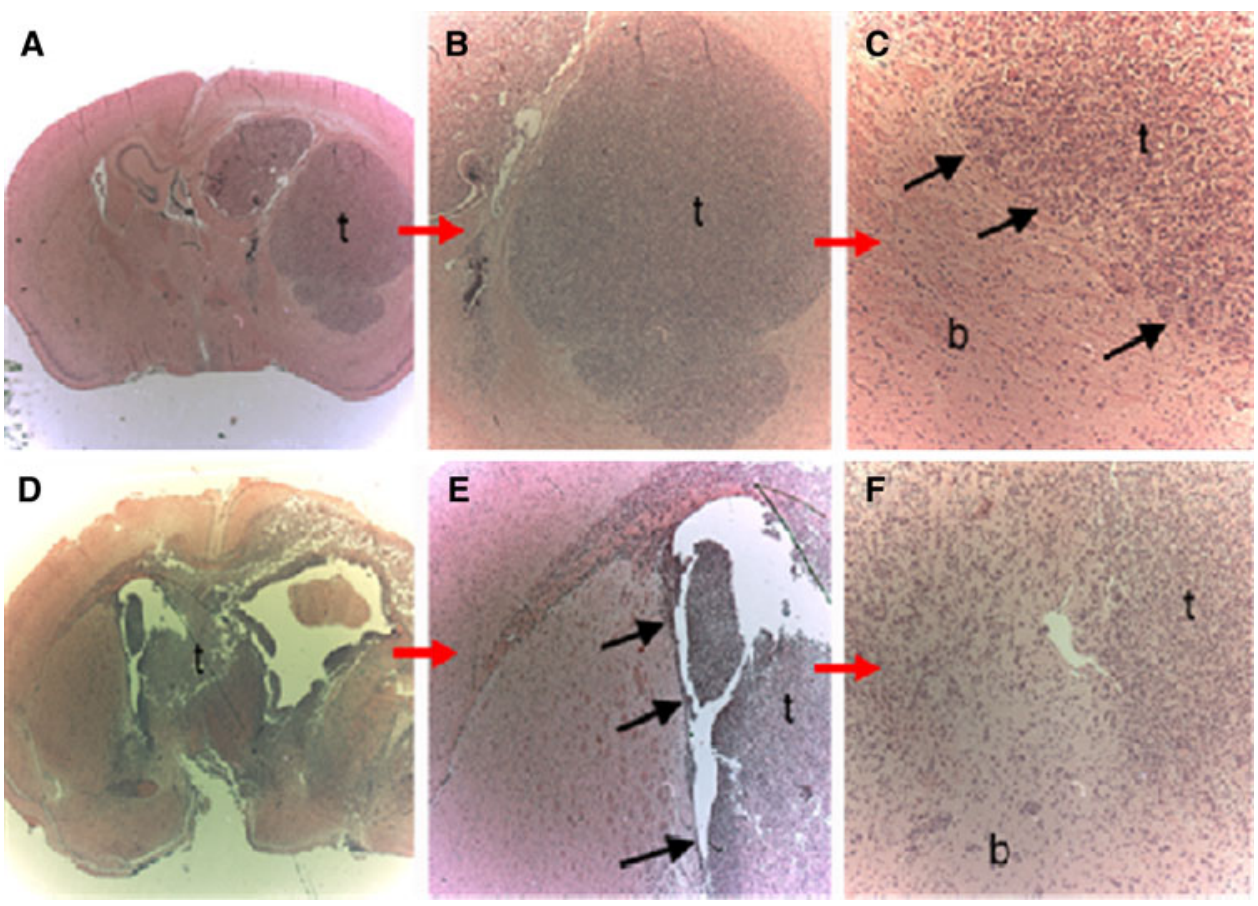

\section{Histopathological correlation}

Histology was performed to confirm the MR findings. The mouse brains ( $n=13: n=6$ U87, $n=7$ hTSC) were removed and sliced to $1 \mathrm{~mm}$ thickness, matching the MR slice thickness. The $1 \mathrm{~mm}$ slices were fixed in formalin, sectioned to $5 \mu \mathrm{m}$, and stained with hematoxylin and eosin (H\&E). The H\&E sections were examined by a pathologist, and cellular atypia, anaplasia, mitotic activity, microvascular proliferation, infiltration and tumor border topography, and necrosis were observed, quantified and tabulated. The tabulated data was then compared to the findings for the corresponding MR slice acquisitions.

\section{MR contrast enhancement measurements}

Brain enhancement represents a measure of tumor microvascular permeability, and relates to how therapeutic agents might be distributed within a tumor and whether contrast agents can highlight a small tumor. We used the usual normalization method in which a ratio of contrast enhancement is determined by comparing tumor tissue to contralateral normal brain. We also calculated a ratio of enhancement comparing the tumor with cervical skeletal muscle (sternocleidomastoid). This was performed to provide a check for the tumor versus normal brain ratio, since it is possible that inaccuracies might arise from altered permeability in the reference white matter due to tumor infiltration and edema.
The tumor to contralateral white matter ratio (TWR) and the tumor-muscle enhancement ratio (TMR) were the mean signal intensity of the enhanced tumor divided by mean signal intensity of white matter and cervical muscle, respectively, at the 12 min post-contrast T1WI. Maximum enhanced regions of interest (ROI) were selected manually on the pre- and post-contrast images by a blinded observer who was not aware of the identities of the scans. The T1 and $\mathrm{T} 2$ acquisitions for each subject were cross-referenced to ensure proper ROI location.

\section{Results}

Tumor morphology imaged by MRI

All the U87 tumors $(n=6)$ had a well-demarcated margin (Fig. 1), and no central necrosis was observed. They had relatively uniform hyperintensity compared to surrounding brain parenchyma on T2WI, and all displayed clearly visible contrast enhancement that was quite uniform. Hydrocephalus often develops with U87 tumors in mice, and was observed in four of the animals. However, no necrotic regions were detected with the U87 tumors. In contrast the hTSC tumors $(n=7)$ exhibited an irregular morphology, and by 6 weeks all the T2WI images displayed variably enhancement regions compared with the uniformity of enhancement seen in U87 tumors. Intratumoral necrosis was not evident on the early scans, but was clearly visible 

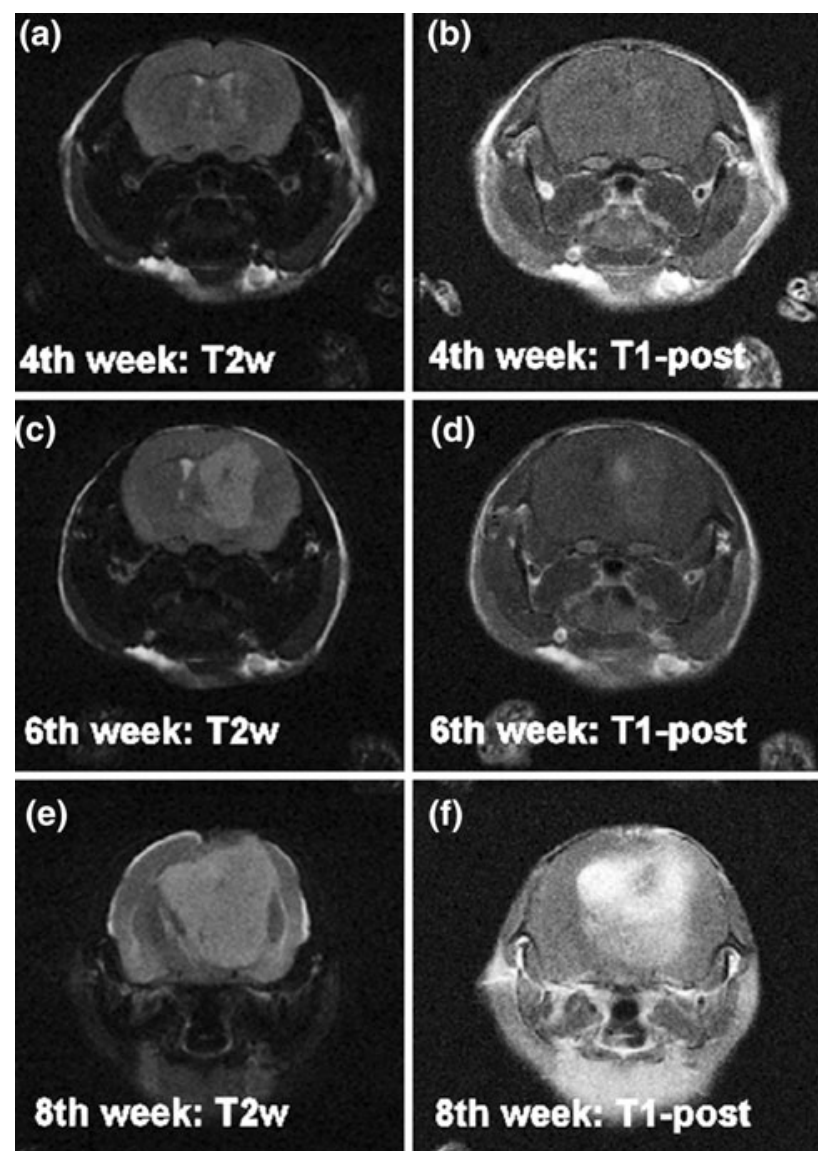

Fig. 3 Longitudinal imaging of hTSC tumors. Four weeks after implantation the hTSC tumors were hyperintense on T2WI (a) and showed post-contrast enhancement in T1WI (b). From the 4th week to 6 th week post-inoculation, the tumor grew from $4.4 \mathrm{~mm}$ diameter (cd) to $6.8 \mathrm{~mm}$ diameter as shown in this T2WI (e-f). Even at 6 weeks the tumor did not enhance as indicated by the post-contrast T1WI (d). By the 8th week the tumor had enlarged significantly as shown by the T2WI acquisition (e), the mouse evinced signs of hydrocephalus, and the post-contrast T1WI image was strongly enhanced

in four mice between 6 and 10 weeks after hTSC implantation. Hydrocephalus due to blockage of the brain ventricles appeared in all hTSC animals by 8 weeks.

\section{Histopathological correlation with MRI morphology}

There was no significant difference between U87 and hTSC tumors in terms of cellular atypia, anaplasia, mitotic activity, and microscopic necrosis. This suggests that failure to detect necrosis in the U87 tumors with MRI may have been due to limitations in MR sensitivity. Notwithstanding, the hTSC tumors were much more irregular that U87 tumors in terms of their overall morphology (Fig. 2). The hTSC tumors showed a very pronounced cellular infiltration of normal brain parenchyma, and were aggressively invasive. Subventricular zone infiltration was present in 6 of 7 hTSC tumors, compared to 0 of 6 of the U87 tumors (Fig. 2a-c). The hTSC tumors very much mimicked human glioma, exhibiting distinct, irregular masses of growth with infiltrative components present to varying degrees in different mice and at different time points.

\section{TMR and TWR contrast enhancement}

In the U87 tumors, which consistently contrast enhanced, the final mean TWR was $1.67 \pm 0.23$ with variance of 13.9, as represented by the maximally enhanced region (Fig. 1). Mean TMR using the same tumor regions was $1.11 \pm 0.06$ with variance of 5.6. Both normalization methods showed similar trends in terms of enhancement, but the TWR was more variable, possibly due to the effects of white matter edema or variations in blood brain barrier permeability and contrast diffusion.

\section{Contrast enhancement}

The hTSC tumors did enhance in 11 of 18 time point measurements $(n=7$ mice), but a significant proportion showed very little or no enhancement at early times. The range for enhancing regions was $8.5-233 \%$. During the development of the hTSC tumors, there was an increase of TWR and TMR across all animals $(n=7)$. A representative example is shown in Fig. 3a showing an abrupt change of vascular permeability. The permeability of all hTSC tumors increased during the course of tumor development and eventually did reach that of U87 tumors (Fig. 4a-c). hTSC tumors exhibited greater variability in microvascular permeability within tumors and across animals than did the U87 tumor group.

MR detection of subtle microvascular effects in hTSC tumors

Our data revealed that minimally enhancing hTSC tumor did in fact show subtle evidence of increased microvascular permeability during the beginning of 2 -week period. Figure 5 shows the MRI characteristics of a representative example from the minimally enhancing glioma. The tumor was very large and had a long T2 (Fig. 5a), but relatively modest enhancement on post-Gadolinium T1WI (Fig. 5b). The invasive tumor tissue (T2WI, Fig. 5c) indicated by the thin white arrows showed very mild enhancement in Fig. 5d. Oval ROIs were drawn on the post-contrast T1WI depicting the location of the cervical muscle reference sites in Fig. $5 \mathrm{~d}$, guided by the T2W image of $5 \mathrm{~d}$. Interestingly, even though the invasive tumor tissue was subtly enhanced, Fig. $5 \mathrm{~d}$ shows that on the corresponding post-contrast T1WI, it was hypointense compared to muscle. In contrast the representative MRI of a U87 tumor in Fig. $5 \mathrm{~g}$ shows that these tumors are highly enhancing compared to muscle 
Fig. 4 Contrast enhancement over time in U87 versus hTSC tumors. a MR image slices acquired for a U87 tumor, at two timepoints separated by 14 days. The upper panels marked 'start' are the first pair of $\mathrm{T} 2 \mathrm{~W}$ and $\mathrm{T} 1 \mathrm{~W}$ post contrast images. For the U87 tumor the initial and final 'end' acquisitions show the same degree of strong enhancement, even though they differ in size. Note the smooth outline of the tumor at 14 days. b MR images, 14 days apart, acquired for an hTSC tumor. The initial acquisition shows little or no enhancement, while the 14 day acquisition has strong enhancement. Note the irregular outline of the tumor in the contrast enhanced image at the second timepoint. c Quantitative representation of contrast enhancement for U87 tumors, for hTSC tumors at beginning of 2-week period 'start' and at conclusion of 2-week period 'end'. The U87 contrast versus hTSC at start of period differed significantly $(P=0.0001$; parametric, unpaired $T$-test), and the start hTSC contrast differed from that at the end $(P=0.0002)$. The mean (line $x)$, the range (box) and the standard deviation (bars) are indicated for each group
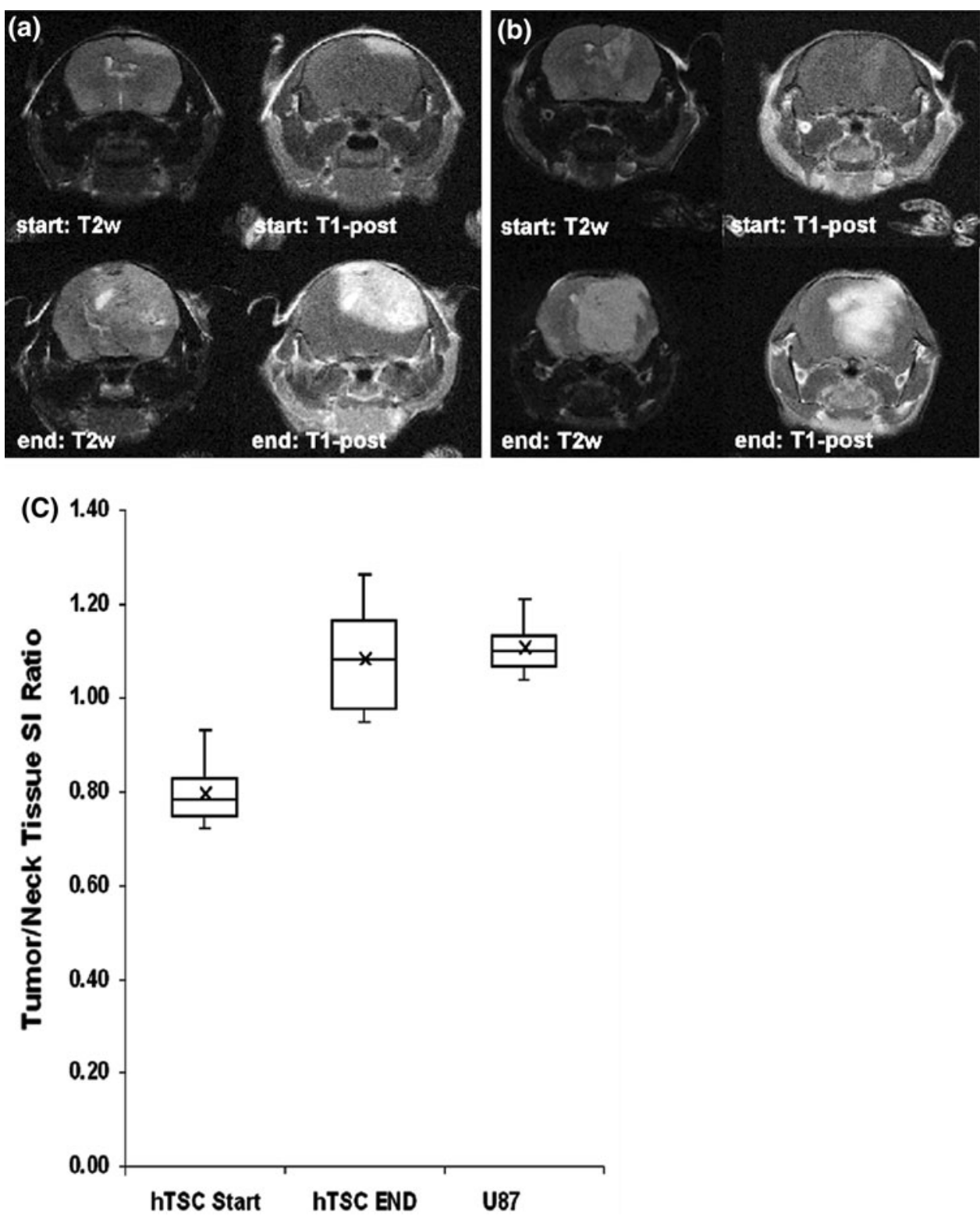

on the delayed post-Gadolinium T1WI. These data reveal that microvascular permeability patterns and changes can be expected to be quite different using the U87 model versus the more physiologically relevant hTSC tumor model.

\section{Discussion}

We report the MR imaging characteristics of a new human stem cell (hTSC) xenograft model [9, 13, 15]. This model was recently developed in response to the generally perceived need for animal models that reproduce the angiogenic and invasive natural history of human glioma $[1,13,15]$. MR imaging is indispensable for managing glioma patients, and MRI has been increasingly used for the assessment of drug effects in animal models of brain cancer [1, 17-19]. Therefore, we sought to complete the hTSC model by characterizing its MR features relative to well-established MR findings in human glioma, and to the widely used U87 glioma model. Conventional MR examinations validated histologically, revealed that hTSC tumors mimicked the MR profile typically observed with glioma patients. The MRIs revealed evidence of angiogenesis and/or increased tumor vessel permeability via longitudinally developing, irregularly distributed contrast enhancement, tumor invasiveness, and heterogeneity 

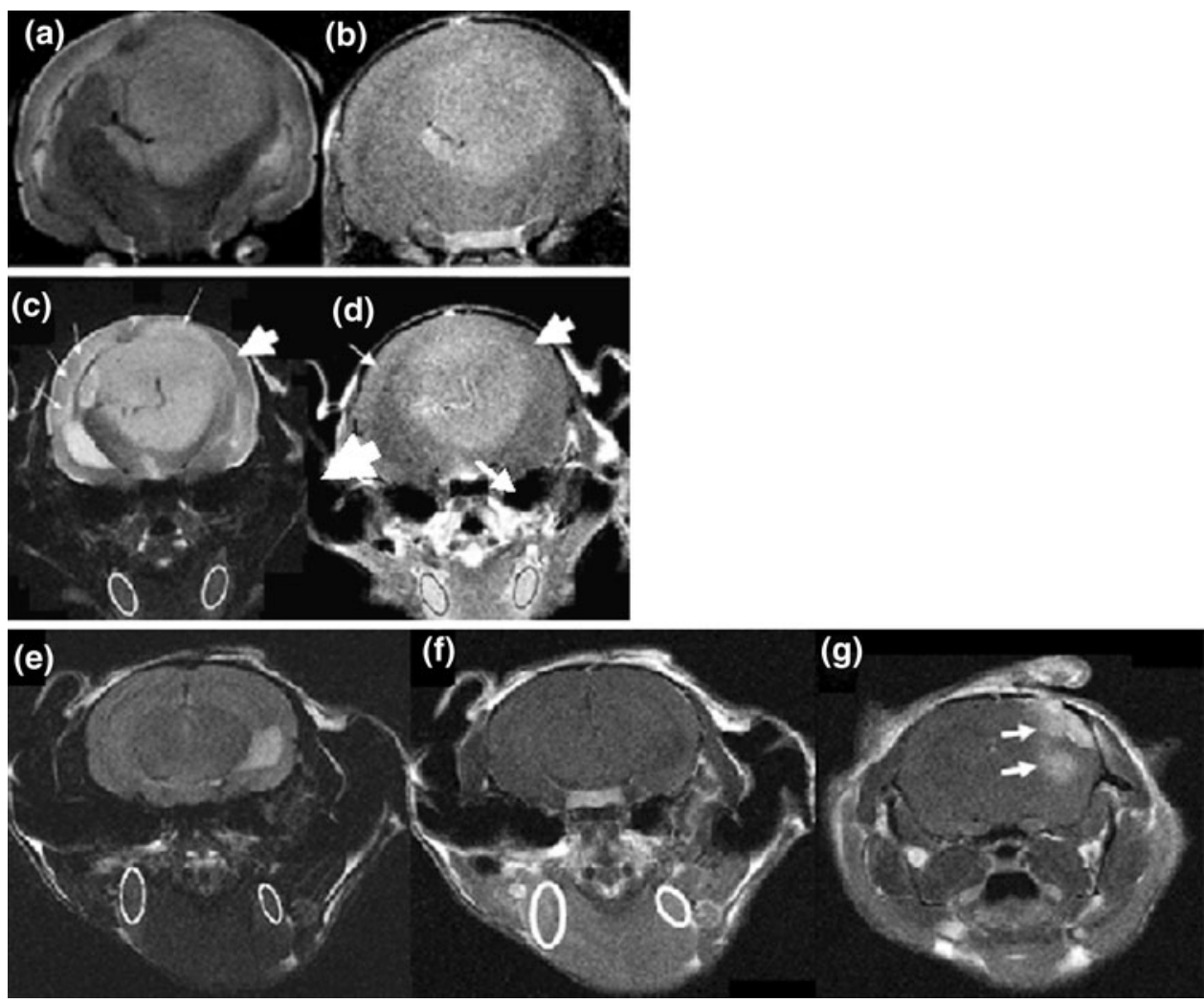

Fig. 5 MR detection of subtle microvascular effects in hTSC tumors. a T2W image of a large hTSC tumor. b Post-contrast T1W image of the same slice; tumor was very large but the contrast enhancement and thus vascular permeability were relatively low. c T2W image used to define cervical muscle ROIs and tumor invasion (thin arrows; thick arrow shows normal tissue). d invasive tumor tissue showing only mild enhancement. Even though the invasive tissue showed subtle enhancement, it was hypointense compared with the cervical muscle reference loci. e T2W image of cervical muscle in a U87 tumor bearing mouse. This image was used to define reference ROIs. f Is the T1W post-contrast image of the same slice as the cervical ROI $\mathrm{T} 2 \mathrm{~W}$ image and shows enhancement in the muscle tissue. g Shows the post-contrast T1W image of the U87 tumor which is clearly hyperintense compared to the cervical muscle and surrounding brain within and between tumors. As expected, the well circumscribed, spherical U87 MR profile and uniform contrast enhancement did not resemble the MR appearance of patient gliomas.

Previous investigations of quantitative permeability imaging in various tumor models has established that tumor enhancement is produced by a combination of: (1) increase in capillary blood volume due to an increase in blood flow within existing brain capillaries, (2) impairment of the blood-brain barrier (BBB) in native capillaries due to diffusible paracrine factors released by the tumor or host immune response, and (3) the formation of new leaky tumor microvessels (angiogenesis), which is the main effect [24, 25]. Thus while Gd detection represents an admixture of effects, escalating Gd enhancement over time is one of the best established clinical surrogate imaging markers of angiogenesis [24, 25]. Low grade human gliomas initially show little contrast enhancement, and then as they progress to higher grade tumors they begin to express strong contrast enhancement [26-30]. On the other hand, high grade gliomas, viz., the glioblastomas, exhibit vivid contrast enhancement upon first presentation, but this is not always the case, as some glioblastomas do not or minimally enhance initially $[31,32]$. Moreover patients are MR imaged after they are symptomatic, so it is possible that early tumors, if they were to be MR imaged, might not enhance until they have progressed sufficiently to cause symptoms. In any case the finding that not all the hTSC tumors enhanced initially, that they all enhanced at 2 weeks, and that they exhibited a heterogeneous and irregular morphology suggests that this model mimics the human disease reasonably well.

Since the hTSC mouse model exhibits a progression from minimal enhancement to full enhancement, it may be an attractive translational test platform for the application of advanced imaging methods to define the temporal evolution of abnormal permeability in infiltrative progressive glioma. The dynamics and kinetics of tumor and brain delivery of therapeutic and contrast agents is considerably influenced by variations in vascular permeability, and based on our results the U87 and hTSC tumors can be expected to behave differently from each 
other in this regard over at least the first few weeks after implantation.

A limitation of the hTSC model is that it involves the implantation of human glioma cells into an athymic $n u / n u$ or SCID mice. These murine models are immunocompromised, and it has been demonstrated that $\mathrm{T}$ cells do occur within gliomas and contribute to their natural history [33].

The abrupt transformation from low enhancement to high enhancement in only the hTSC model, documents the sensitivity range of MRI in this model. This event is thought to result from the release of angiogenic factors and matrix metalloproteinases, and in humans heralds the onset of an angiogenic, aggressive glioma phenotype [8, 28, 30]. Future work on this topic will require a comprehensive study in which the tumors are staged, and vascular permeability and density around the tumor are characterized using MR functional and histological approaches. This is beyond the scope of the present study, but a further systematic examination of the possible relationship between suddenly increased MR contrast enhancement in hTSC tumors and the angiogenesis will be the subject of a future research.

Acknowledgments The authors gratefully acknowledge support from the Functional and Molecular Imaging Center of Department of Radiology, Brigham and Women's Hospital and the previous Center for Bioinformatics, Harvard Center for Neurodegeneration and Repair (now Harvard Neurodiscovery Center), Harvard Medical School (KW, KC, STCW), and the Brain Tumor Clinical Research Fund. This work was supported in part by grants from NIH K08CA124804, ARRA 3P30CA023100-25S8, Sontag Foundation Distinguished Scientist Award, and a James S. McDonnell Foundation award to SK.

Open Access This article is distributed under the terms of the Creative Commons Attribution Noncommercial License which permits any noncommercial use, distribution, and reproduction in any medium, provided the original author(s) and source are credited.

\section{References}

1. Becher OJ, Holland EC (2006) Genetically engineered models have advantages over xenografts for preclinical studies. Cancer Res 66(7):3355-3359

2. Winkler F, Kienast Y, Fuhrmann M, Von Baumgarten L, Burgold S, Mitteregger G, Kretzschmar H, Herms J (2009) Imaging glioma cell invasion in vivo reveals mechanisms of dissemination and peritumoral angiogenesis. Glia 57(12):1306-1315

3. Stupp R, Hegi ME, Gilbert MR, Chakravarti A (2007) Chemoradiotherapy in malignant glioma: standard of care and future directions. J Clin Oncol 25(26):4127-4136

4. Lorger M, Krueger JS, O’Neal M, Staflin K, Felding-Habermann B (2009) Activation of tumor cell integrin alphavbeta3 controls angiogenesis and metastatic growth in the brain. Proc Natl Acad Sci USA 106(26):10666-10671

5. Szentirmai O, Baker CH, Lin N, Szucs S, Takahashi M, Kiryu S, Kung AL, Mulligan RC, Carter BS (2006) Noninvasive bioluminescence imaging of luciferase expressing intracranial U87 xenografts: correlation with magnetic resonance imaging determined tumor volume and longitudinal use in assessing tumor growth and antiangiogenic treatment effect. Neurosurgery 58(2):365-372

6. Koutcher JA, Hu X, Xu S, Gade TP, Leeds N, Zhou XJ, Zagzag D, Holland EC (2002) MRI of mouse models for gliomas shows similarities to humans and can be used to identify mice for preclinical trials. Neoplasia 4(6):480-485

7. McConville P, Hambardzumyan D, Moody JB, Leopold WR, Kreger AR, Woolliscroft MJ, Rehemtulla A, Ross BD, Holland EC (2007) Magnetic resonance imaging determination of tumor grade, early response to temozolomide in a genetically engineered mouse model of glioma. Clin Cancer Res 13(10): 2897-2904

8. Ligon KL, Huillard E, Mehta S, Kesari S, Liu H, Alberta JA, Bachoo RM, Kane M, Louis DN, Depinho RA, Anderson DJ, Stiles CD, Rowitch DH (2007) Olig2-regulated lineage-restricted pathway controls replication competence in neural stem cells and malignant glioma. Neuron 53(4):503-517

9. Norden AD, Young GS, Setayesh K, Muzikansky A, Klufas R, Ross GL, Ciampa AS, Ebbeling LG, Levy B, Drappatz J, Kesari S, Wen PY (2008) Bevacizumab for recurrent malignant gliomas: efficacy, toxicity, and patterns of recurrence. Neurology 70(10): 779-787

10. Jost SC, Wanebo JE, Song SK, Chicoine MR, Rich KM, Woolsey TA, Lewis JS, Mach RH, Xu J, Garbow JR (2007) In vivo imaging in a murine model of glioblastoma. Neurosurgery 60(2):360-370

11. Sun Y, Schmidt NO, Schmidt K, Doshi S, Rubin JB, Mulkern RV, Carroll R, Ziu M, Erkmen K, Poussaint TY, Black P, Albert M, Burstein D, Kieran MW (2004) Perfusion MRI of U87 brain tumors in a mouse model. Magn Reson Med 51(5):893-899

12. Li A, Walling J, Kotliarov Y, Center A, Steed ME, Ahn SJ, Rosenblum M, Mikkelsen T, Zenklusen JC, Fine HA (2008) Genomic changes and gene expression profiles reveal that established glioma cell lines are poorly representative of primary human gliomas. Mol Cancer Res 6(1):21-30

13. Lee J, Kotliarova S, Kotliarov Y, Li A, Su Q, Donin NM, Pastorino S, Purow BW, Christopher N, Zhang W, Park JK, Fine HA (2006) Tumor stem cells derived from glioblastomas cultured in bFGF and EGF more closely mirror the phenotype and genotype of primary tumors than do serum-cultured cell lines. Cancer Cell 9(5):391-403

14. Martinez-Murillo R, Martinez A (2007) Standardization of an orthotopic mouse brain tumor model following transplantation of CT-2A astrocytoma cells. Histol Histopathol 22(12):1309

15. Bachoo RM, Maher EA, Ligon KL, Sharpless NE, Chan SS, You MJ, Tang Y, DeFrances J, Stover E, Weissleder R, Rowitch DH, Louis DN, DePinho RA (2002) Epidermal growth factor receptor and Ink4a/Arf: convergent mechanisms governing terminal differentiation and transformation along the neural stem cell to astrocyte axis. Cancer Cell 1(3):269-277

16. Dass CR, Choong PF (2007) GFP expression alters osteosarcoma cell biology. DNA Cell Biol 26(8):599-601

17. Castillo M, Smith JK, Kwock L, Wilber K (2001) Apparent diffusion coefficients in the evaluation of high-grade cerebral gliomas. AJNR Am J Neuroradiol 22(1):60-64

18. Nelson SJ, Cha S (2003) Imaging glioblastoma multiforme. Cancer J 9(2):134-145

19. Sugahara T, Korogi Y, Kochi M, Ikushima I, Hirai T, Okuda T, Shigematsu Y, Liang L, Ge Y, Ushio Y, Takahashi M (1998) Correlation of MR imaging-determined cerebral blood volume maps with histologic and angiographic determination of vascularity of gliomas. AJR Am J Roentgenol 171(6):1479-1486

20. Barker FG 2nd, Chang SM, Huhn SL, Davis RL, Gutin PH, McDermott MW, Wilson CB, Prados MD (1997) Age and the 
risk of anaplasia in magnetic resonance-nonenhancing supratentorial cerebral tumors. Cancer 80(5):936-941

21. Ginsberg LE, Fuller GN, Hashmi M, Leeds NE, Schomer DF (1998) The significance of lack of MR contrast enhancement of supratentorial brain tumors in adults: histopathological evaluation of a series. Surg Neurol 49(4):436-440

22. Atkinson M, Juhasz C, Shah J, Guo X, Kupsky W, Fuerst D, Johnson R, Watson C (2008) Paradoxical imaging findings in cerebral gliomas. J Neurol Sci 269(1-2):180-183

23. Stockhammer F, Plotkin M, Amthauer H, van Landeghem FK, Woiciechowsky C (2008) Correlation of F-18-fluoro-ethyl-tyrosin uptake with vascular and cell density in non-contrastenhancing gliomas. J Neurooncol 88(2):205-210

24. Gagner JP, Law M, Fischer I, Newcomb EW, Zagzag D (2005) Angiogenesis in gliomas: imaging and experimental therapeutics. Brain Pathol 15(4):342-363

25. Gossmann A, Helbich TH, Kuriyama N, Ostrowitzki S, Roberts TP, Shames DM, van Bruggen N, Wendland MF, Israel MA, Brasch RC (2002) Dynamic contrast-enhanced magnetic resonance imaging as a surrogate marker of tumor response to antiangiogenic therapy in a xenograft model of glioblastoma multiforme. J Magn Reson Imaging 15(3):233-240

26. Kenney J, Schmiedl U, Maravilla K, Starr F, Graham M, Spence A, Nelson J (1992) Measurement of blood-brain barrier permeability in a tumor model using magnetic resonance imaging with gadolinium-DTPA. Magn Reson Med 27(1):68-75
27. Zagzag D, Amirnovin R, Greco MA, Yee H, Holash J, Wiegand SJ, Zabski S, Yancopoulos GD, Grumet M (2000) Vascular apoptosis and involution in gliomas precede neovascularization: a novel concept for glioma growth and angiogenesis. Laboratory Investig 80(6):837-849

28. Reardon DA, Wen PY, Desjardins A, Batchelor TT, Vredenburgh JJ (2008) Glioblastoma multiforme: an emerging paradigm of anti-VEGF therapy. Expert Opin Biol Ther 8(4):541-553

29. Abdollahi A, Schwager C, Kleeff J, Espositos I, Domhan S, Peschke $\mathrm{P}$ et al (2007) Transcriptional network governing the angiogenic switch in human pancreatic cancer. Proc Natl Acad Sci USA 104(31):12890-12895

30. Roberts HC, Roberts TP, Brasch RC, Dillon WP (2000) Quantitative measurement of microvascular permeability in human brain tumors achieved using dynamic contrast-enhanced MR imaging: correlation with histologic grade. AJNR Am J Neuroradiol 21(5):891-899

31. Landy HJ, Lee TT, Potter P, Feun L, Markoe A (2000) Early MRI findings in high grade glioma. J Neurooncol 47:65-72

32. Cha S (2005) Diagnostic imaging. Section 1B, Diagnosis. In: Berger MS, Prados MD (eds) Textbook of neuro-oncology. Elsevier Saunders, Philadelphia

33. Sonabend AM, Rolle CE, Lesniak MS (2008) The role of regulatory $\mathrm{T}$ cells in malignant glioma. Anticancer Res 28(2B): $1143-1150$ 